\title{
A Homotopy-Analysis Approach for Nonlinear Wave-Like Equations with Variable Coefficients
}

\author{
Afgan Aslanov \\ Mathematics and Computing Department, Beykent University, 34396 Istanbul, Turkey \\ Correspondence should be addressed to Afgan Aslanov; afganaslanov@beykent.edu.tr
}

Received 20 February 2015; Accepted 23 April 2015

Academic Editor: R. Naz

Copyright (C) 2015 Afgan Aslanov. This is an open access article distributed under the Creative Commons Attribution License, which permits unrestricted use, distribution, and reproduction in any medium, provided the original work is properly cited.

We are interested in the approximate analytical solutions of the wave-like nonlinear equations with variable coefficients. We use a wave operator, which provides a convenient way of controlling all initial and boundary conditions. The proposed choice of the auxiliary operator helps to find the approximate series solution without any discretization, linearization, or restrictive assumptions. Several examples are given to verify the reliability and efficiency of the method.

\section{Introduction}

We consider the equation

$$
\begin{aligned}
u_{t t}-k(x, t) u_{x x}=f\left(u, u_{x}, u_{y}, u_{x t}\right)+g(x, t) & \\
x & >0, t>0
\end{aligned}
$$

with initial conditions

$$
\begin{aligned}
u(x, 0) & =\varphi(x), \\
u_{t}(x, 0) & =\psi(x)
\end{aligned}
$$

and boundary condition

$$
u(0, t)=h(t)
$$

where $f, g, k, \varphi, \psi$, and $h$ are known functions.

Note that the proposed method can be applied for equations like

$$
k(x, t) u_{t t}-u_{x x}=f\left(u, u_{x}, u_{y}, u_{x t}\right)+g(x, t),
$$

with the same type of initial-boundary conditions.

Problems like (1)-(3) model many problems in classical and quantum mechanics, solitons, and matter physics $[1,2]$. If $f$ is a function of $u$ only and $k(x, t)=$ const we obtain KleinGordon or sine-Gordon type equations. These models can describe some nonlinear phenomena; for example, wave-like equation can describe earthquake stresses [3], coupling currents in a flat multistrand two-layer superconducting cable [4], and nonhomogeneous elastic waves in soils [5]. Typical examples of the wave-like equations with variable coefficients are Euler-Tricomi equation [6] or Chaplygin equation [7] given by

$$
\begin{aligned}
u_{t t}-t u_{x x} & =0, \\
u_{t t}-k(t) u_{x x} & =0,
\end{aligned}
$$

which are useful in the study of transonic flow, where $u=$ $u(t, x)$ is the stream function of a plane-parallel steady-state gas flow, $k(t)$ is positive at subsonic and negative at supersonic speed, and $x$ is the angle of inclination of the velocity vector. Some Chaplygin type of equation of the special form

$$
u_{t t}+\frac{x^{2}}{1-x^{2} / c^{2}} u_{x x}+x u_{x}=0,
$$

where $c=c(x)$ is the speed of sound, has also applications in the study of transonic flow [8].

Note that we use the term wave-like equation to describe the partial differential equations with the terms $u_{t t}$ and $u_{x x}$; that is, the term "wave-like" may not correspond to the real physical waves, in general.

Recently, there has been a growing interest for obtaining the explicit solutions to wave-like and heat-like models by 
analytic techniques. Wazwaz [9] used the tanh method to obtain the exact solution of the sine-Gordon equation. Kaya [10] applied the modified decomposition method to solve the sine-Gordon equation. Aslanov [11] used homotopy perturbation method to solve Klein-Gordon type of equations with unbounded right-hand side. El-Sayed [12] and Wazwaz and Gorguis [13] used Adomian decomposition method for solving wave-like and heat-like problems.

The homotopy analysis method [14-18] is developed to search the accurate asymptotic solutions of nonlinear problems. Liao [17] proved that the homotopy analysis method (HAM) contains some other nonperturbation techniques, such as Adomian's decomposition method and Lyapunov's artificial small parameter. Hayat and Sajid [19] and Abbasbandy [20] pointed out that the homotopy perturbation method is only a special case of the HAM. Aslanov [21] used homotopy perturbation method to solve wave-like equations with initial-boundary conditions. Rajaraman [22] and Alomari at al. [23] applied HAM for solving nonlinear equations with initial conditions. Öziş and Ağırseven [24] used homotopy perturbation method for solving heat-like and wave-like equations with variable coefficients.

Various methods for obtaining exact and approximate solutions to nonlinear partial differential equations have been proposed. Among these methods are the homotopy perturbation and Adomian decomposition methods [25-29], the variational iteration method [30], homotopy analysis method [31], and others.

Here we will further extend the applications of HAM to obtain an approximate series solution for the nonlinear wavelike equations with variable coefficients and with initialboundary conditions. The difficulty in the use of standard HAM is that the choice of the linear operator $L$ in standard form (like $L u=u_{t t}$ or $L u=u_{x x}$ ) cannot control the boundary conditions (2)-(3).

Unlike the various approximation techniques, which are usually valid for problems with (only) initial conditions, our technique is applicable for a wide range of initial-boundary problems of types (1)-(3). The central idea here is that the problem,

$$
\begin{aligned}
u_{t t}-c^{2} u_{x x} & =g(x, t), \\
u(x, 0) & =\varphi(x), \\
u_{t}(x, 0) & =\psi(x), \\
u(0, t) & =h(t),
\end{aligned}
$$

has a unique solution (see, e.g., [32]) and therefore there exists an inverse of the operator $L: u_{t t}-c^{2} u_{x x}$. This operator can control all initial-boundary conditions in each step of HAM.

Therefore we rewrite (1) as

$$
\begin{aligned}
L u= & -c^{2} u_{x x}+k(x, t) u_{x x}+f\left(u, u_{x}, u_{y}, u_{x y}\right) \\
& +g(x, t),
\end{aligned}
$$

for some appropriate constant $c$, and construct the so-called zero-order deformation equation $[14,15]$ :

$$
(1-q) L\left[\phi(x, t ; q)-u_{0}(x, t)\right]=q \hbar N[\phi(x, t ; q)],
$$

where $q \in[0,1]$ is an embedding parameter, $\hbar$ is a nonzero auxiliary function, $L(u)=u_{t t}-c^{2} u_{x x}, N(\phi)=\phi_{t t}-$ $k(x, t) \phi_{x x}-f\left(\phi, \phi_{x}, \phi_{y}, \phi_{x t}\right)-g(x, t), u_{0}(x, t)$ is an initial guess, and $\phi(x, t ; q)$ is an unknown function. The conditions $q=0$ and $q=1$ correspond to

$$
\begin{aligned}
& \phi(x, t ; 0)=u_{0}(x, t), \\
& \phi(x, t ; 1)=u(x, t),
\end{aligned}
$$

respectively. Thus as $q$ increases from 0 to 1 , the solution $\phi(x, t ; q)$ varies from the initial guess $u_{0}(x, t)$ to the solution $u(x, t)[14,15]$.

Expanding $\phi(x, t ; q)$ in Taylor series with respect to $q$, one has

$$
\phi(x, t ; q)=u_{0}(x, t)+\sum_{m=1}^{\infty} u_{m}(x, t) q^{m}
$$

where

$$
u_{m}(x, t)=\left.\frac{1}{m !} \frac{\partial^{m} \phi(x, t, q)}{\partial q^{m}}\right|_{q=0}
$$

If the auxiliary linear operator, the initial guess, the auxiliary parameter $\hbar$, and the auxiliary function are so properly chosen, then series (11) converges at $q=1$ and

$$
\phi(x, t ; 1)=u_{0}(x, t)+\sum_{m=1}^{\infty} u_{m}(x, t)
$$

which must be one of solutions of the original equation, as proved by Liao [17].

According to definition (12), the governing equation can be deduced from the zero-order deformation equation (9). Define the vector

$$
\vec{u}_{n}=\left\{u_{0}, u_{1}, \ldots, u_{n}\right\}
$$

Differentiating equation (9) $m$ times with respect to embedding parameter $q$ and then setting $q=0$, we have the so-called $m$ th-order deformation equation

$$
L\left[u_{m}-\chi_{m} u_{m-1}\right]=\hbar R_{m}\left(\vec{u}_{m-1}\right)
$$

where

$$
\begin{aligned}
R_{m}\left(\vec{u}_{m-1}\right) & =\left.\frac{1}{(m-1) !} \frac{\partial^{m-1} N(\phi(x, t, q))}{\partial q^{m-1}}\right|_{q=0}, \\
\chi_{m} & = \begin{cases}0, & m \leq 1 \\
1, & m>1 .\end{cases}
\end{aligned}
$$

\section{Applications}

To demonstrate the advantages of our approach first we consider the wave-like linear equation with constant coefficients. 
Example 1. We consider the initial-boundary value problem

$$
\begin{aligned}
u_{t t}+u_{x x} & =-2 u_{x t}, \quad x>0, t>0, \\
u(x, 0) & =x^{3}, \\
u_{t}(x, 0) & =-3 x^{2}, \\
u(0, t) & =t^{3} .
\end{aligned}
$$

The exact solution is $u=\left|(x-t)^{3}\right|$.

The traditional methods (with $L u=u_{t t} \cdots$ ) do not work for this kind of problems. For example, the operator $L u=u_{t t}$ can not control the condition $u(0, t)=0$ in every iteration step, the same for the operator $L u=u_{x x}$; that is, we need an operator that can control all initial/boundary conditions. Clearly the most appropriate operator should be the wave operator. We take $L(\phi)=\partial^{2} \phi(x, t, q) / \partial t^{2}-\partial^{2} \phi(x, t, q) / \partial x^{2}$. Equation (1) suggests that we define the nonlinear operator as

$$
N(\phi)=2 \frac{\partial^{2} \phi(x, t, q)}{\partial x^{2}}+2 \frac{\partial^{2} \phi(x, t, q)}{\partial x \partial t} .
$$

Using the above definition, we construct the zeroth-order deformation equation

$$
(1-q) L\left[\phi(x, t ; q)-u_{0}(x, t)\right]=q \hbar N[\phi(x, t ; q)]
$$

and the $m$ th order deformation equation

$$
L\left[u_{m}-\chi_{m} u_{m-1}\right]=\hbar R_{m}\left(\vec{u}_{m-1}\right),
$$

with the initial/boundary conditions $u_{m}(x, 0)=0$, $\left(u_{m}\right)_{t}(x, 0)=0$, and $u_{m}(0, t)=0$.

Now it follows from the theory of wave equations that [32] the solution of the equation $u_{t t}-u_{x x}=0$ with the same initialboundary conditions is

$$
\begin{aligned}
u_{0}(x, t)= & \frac{1}{2}\left((x+t)^{3}+(x-t)^{3}\right)-\frac{1}{2} \int_{x-t}^{x+t}\left(3 y^{2}\right) d y \\
= & (x-t)^{3} \quad \text { for } x>t \\
u_{0}(x, t)= & \frac{1}{2}\left((x+t)^{3}+(x-t)^{3}\right)-\frac{1}{2} \int_{t-x}^{x+t}\left(3 y^{2}\right) d y \\
& +(t-x)^{3}=(t-x)^{3}, \quad \text { for } x<t
\end{aligned}
$$

According to (21) we now successively obtain

$$
L\left(u_{1}\right)=\hbar\left[\left(2 u_{0}\right)_{x x}+\left(2 u_{0}\right)_{x t}\right] \equiv g(x, t)=0
$$

and it follows from

$$
\begin{aligned}
u_{1}= & \frac{\hbar}{2} \int_{0}^{t} \int_{x-t+s}^{x+t-s} g(y, s) d y d s, \quad \text { for } x>t, \\
u_{1}= & \frac{\hbar}{2} \int_{0}^{t-x} \int_{t-x-s}^{x+t-s} g(y, s) d y d s \\
& +\frac{\hbar}{2} \int_{t-x}^{t} \int_{x-t+s}^{x+t-s} g(y, s) d y d s, \text { for } x<t
\end{aligned}
$$

that $u_{1}=u_{2}=\cdots=0[32]$.
Example 2. We consider the initial-boundary value problem

$$
\begin{aligned}
u_{t t} & =\frac{6-(x-t)^{2}}{6} u_{x x}+u, \quad x>0, t>0, \\
u(x, 0) & =x^{3}, \\
u_{t}(x, 0) & =-3 x^{2}, \\
u(0, t) & =0 .
\end{aligned}
$$

The exact solution is

$$
u= \begin{cases}(x-t)^{3} & \text { if } x>t \\ 0 & \text { if } x<t\end{cases}
$$

First we rewrite the equation as

$$
u_{t t}-u_{x x}=\frac{-(x-t)^{2}}{6} u_{x x}+u
$$

The solution of the equation $u_{t t}-u_{x x}=0$ with the same initial-boundary conditions will be taken as an initial approximation:

$$
\begin{aligned}
u_{0} & =\frac{1}{2}\left((x+t)^{3}+(x-t)^{3}\right)-\frac{1}{2} \int_{x-t}^{x+t} 3 y^{2} d y \\
& =(x-t)^{3} \quad \text { for } x>t, \\
u_{0} & =\frac{1}{2}\left((x+t)^{3}-(t-x)^{3}\right)-\frac{1}{2} \int_{t-x}^{x+t} 3 y^{2} d y=0
\end{aligned}
$$

for $x<t$.

For $u_{1}$ we have

$$
L\left(u_{1}\right)=\hbar\left[\frac{(x-t)^{2}}{6} u_{0 x x}-u_{0}\right]
$$

and therefore

$$
\begin{aligned}
& u_{1} \\
& =\frac{\hbar}{2} \int_{0}^{t} \int_{x-t+s}^{x+t-s}\left(\frac{(y-s)^{2}}{6} 6(y-s)-(y-s)^{3}\right) d y d s \\
& =0, \quad \text { for } x>t, \\
& u_{1} \\
& =\frac{\hbar}{2} \int_{0}^{t-x} \int_{t-x-s}^{x+t-s}(0) d y d s+\frac{\hbar}{2} \int_{t-x}^{t} \int_{x-t+s}^{x+t-s}(0) d y d s \\
& =0, \quad \text { for } x<t,
\end{aligned}
$$

and continuing in this way, we obtain $u_{2}=u_{3}=\cdots=0$ and $u_{\text {exact }}=u_{0}+u_{1}$.

The above example demonstrates the importance of the proposed technique with the use of wave operator. In fact, all traditional approaches with some auxiliary operator 
TABLE 1: Maximum errors for Example 3.

\begin{tabular}{lcccccccc}
\hline$t \backslash x$ & 0.1 & 0.2 & 0.3 & 0.4 & 0.5 & 0.6 & 0.8 \\
\hline 0.1 & $9.0 \cdot 10^{-6}$ & $9.0 \cdot 10^{-6}$ & $9.0 \cdot 10^{-6}$ & $9.0 \cdot 10^{-6}$ & $9.0 \cdot 10^{-6}$ & $9.0 \cdot 10^{-6}$ & $9.0 \cdot 10^{-6}$ & $9.0 \cdot 10^{-6}$ \\
0.2 & $1.4 \cdot 10^{-4}$ & $1.5 \cdot 10^{-4}$ & $1.5 \cdot 10^{-4}$ & $1.5 \cdot 10^{-4}$ & $1.5 \cdot 10^{-4}$ & $1.5 \cdot 10^{-4}$ & $1.5 \cdot 10^{-4}$ & $1.5 \cdot 10^{-4}$ \\
0.4 & $2.0 \cdot 10^{-3}$ & $2.7 \cdot 10^{-3}$ & $2.9 \cdot 10^{-3}$ & $2.9 \cdot 10^{-3}$ & $2.9 \cdot 10^{-3}$ & $2.9 \cdot 10^{-3}$ & $2.9 \cdot 10^{-3}$ & $2.9 \cdot 10^{-3}$ \\
0.5 & $4.7 \cdot 10^{-3}$ & $6.8 \cdot 10^{-3}$ & $7.5 \cdot 10^{-3}$ & $7.6 \cdot 10^{-3}$ & $7.6 \cdot 10^{-3}$ & $7.6 \cdot 10^{-3}$ & $7.6 \cdot 10^{-3}$ & $7.6 \cdot 10^{-3}$ \\
0.7 & $1.7 \cdot 10^{-2}$ & $2.6 \cdot 10^{-2}$ & $3.1 \cdot 10^{-2}$ & $3.3 \cdot 10^{-2}$ & $3.4 \cdot 10^{-2}$ & $3.4 \cdot 10^{-2}$ & $3.4 \cdot 10^{-2}$ & $3.4 \cdot 10^{-2}$ \\
0.8 & $2.9 \cdot 10^{-2}$ & $4.6 \cdot 10^{-2}$ & $5.5 \cdot 10^{-2}$ & $6.0 \cdot 10^{-2}$ & $6.2 \cdot 10^{-2}$ & $6.3 \cdot 10^{-2}$ & $6.3 \cdot 10^{-2}$ & $6.3 \cdot 10^{-2}$ \\
0.9 & $4.6 \cdot 10^{-2}$ & $7.5 \cdot 10^{-2}$ & $9.2 \cdot 10^{-2}$ & 0.1020 & 0.10682 & 0.10888 & 0.10973 & 0.10974 \\
\hline
\end{tabular}

$L u=u_{t t}$ do not work, since the (exact) solution is not analytical function on the whole region. For example, the approaches used in $[23,24]$ can not be applied to solve this problem (note that the approaches used in $[23,24]$ are effective and simple for the problems with analytical solutions and/or for the problems with initial conditions).

Example 3. We consider the nonlinear initial-boundary value problem

$$
\begin{aligned}
u_{t t}-x^{2} u_{x x} & =x e^{t}-\left(u_{x}\right)^{2}+e^{2 t}, \\
u(x, 0) & =x, \\
u_{t}(x, 0) & =x, \\
u(0, t) & =0 .
\end{aligned}
$$

The exact solution is $u=x e^{t}$. We take $L(u)=u_{t t}-u_{x x}$ and $u_{0}$ as a solution of the problem $L(u)=0$ with the initial/ boundary conditions $u(x, 0)=x, u_{t}(x, 0)=x$, and $u(0, t)=$ 0 [32]:

$$
\begin{aligned}
& u_{0}=\frac{1}{2}[(x+t)+(x-t)]+\frac{1}{2} \int_{x-t}^{x+t} y d y=x(t+1) \\
& \quad \text { for } x>t, \\
& u_{0}=\frac{1}{2}[(x+t)-(t-x)]+\frac{1}{2} \int_{t-x}^{x+t} y d y=x(t+1)
\end{aligned}
$$

And now we obtain from (16)

$$
\begin{aligned}
L\left(u_{1}\right)= & -\hbar\left[-\left(1-x^{2}\right)\left(u_{0}\right)_{x x}+x e^{t}-\left(u_{0 x}\right)^{2}+e^{2 t}\right] \\
= & -\hbar\left[x e^{t}-(t+1)^{2}+e^{2 t}\right], \\
u_{1}= & -\frac{\hbar}{2} \int_{0}^{t} \int_{x-t+s}^{x+t-s} g(y, s) d y d s, \quad \text { for } x>t, \\
u_{1}= & -\frac{\hbar}{2} \int_{0}^{t-x} \int_{t-x-s}^{x+t-s} g(y, s) d y d s \\
& -\frac{\hbar}{2} \int_{t-x}^{t} \int_{x-t+s}^{x+t-s} g(y, s) d y d s \quad \text { for } x<t,
\end{aligned}
$$

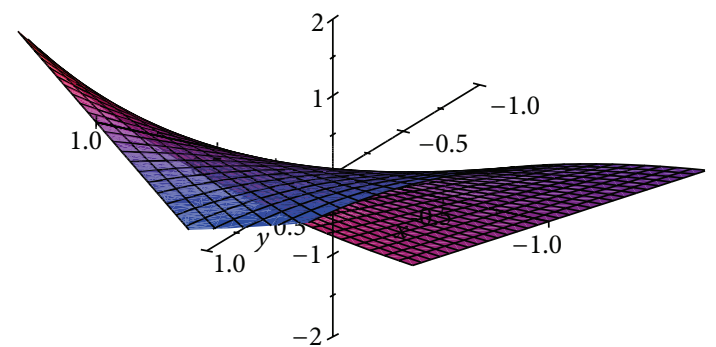

Figure 1: The exact solution for Example 3.

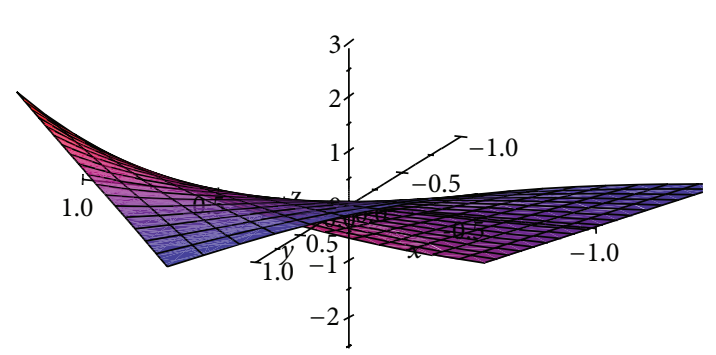

FIGURE 2: The approximate solution for Example 3.

where $g(y, s)=y e^{s}-(s+1)^{2}+e^{2 s}$. Hence we obtain

$$
u_{1}=-\hbar\left(x e^{t}-x-t x-\frac{1}{2} t-\frac{1}{2} t^{2}-\frac{1}{3} t^{3}-\frac{1}{12} t^{4}\right.
$$$$
\left.+\frac{1}{4} e^{2 t}-\frac{1}{4}\right), \quad \text { for } x>t \text {, }
$$

$$
\begin{aligned}
u_{1} & =-\hbar\left(t x^{2}-\frac{3 x}{2}-t^{2} x-\frac{t x^{3}}{3}-\frac{t^{3} x}{3}+\frac{t^{2} x^{2}}{2}-\frac{e^{2 t}}{4 e^{2 x}}\right. \\
& \left.-2 t x+x e^{t}+\frac{x^{2}}{2}-\frac{x^{3}}{3}+\frac{x^{4}}{12}+\frac{e^{2 t}}{4}\right)
\end{aligned}
$$

for $x<t$. The absolute errors between the exact and the twoterm approximation of the series solution for some values of $(x, t) \in[0,1] \times[0,1]$ with $\hbar=-1$ are shown in Table 1. The exact solution for Example 3 is shown in Figure 1 and the approximate solution is shown in Figure 2. A higher accuracy level can be attained by evaluating some more terms. 
TABLE 2: Maximum errors for Example 4.

\begin{tabular}{lcccccccc}
\hline$t \backslash x$ & 0.1 & 0.2 & 0.3 & 0.4 & 0.5 & 0.6 & 0.8 & 0.9 \\
\hline 0.1 & $3.2 \cdot 10^{-4}$ & $3.3 \cdot 10^{-4}$ & $3.3 \cdot 10^{-4}$ & $3.3 \cdot 10^{-4}$ & $3.3 \cdot 10^{-4}$ & $3.3 \cdot 10^{-4}$ & $3.3 \cdot 10^{-4}$ & $3.3 \cdot 10^{-4}$ \\
0.2 & $1.2 \cdot 10^{-3}$ & $2.6 \cdot 10^{-3}$ & $2.6 \cdot 10^{-3}$ & $2.6 \cdot 10^{-3}$ & $2.6 \cdot 10^{-3}$ & $2.6 \cdot 10^{-3}$ & $2.6 \cdot 10^{-3}$ & $2.6 \cdot 10^{-3}$ \\
0.4 & $3.3 \cdot 10^{-3}$ & $6.6 \cdot 10^{-3}$ & $1.4 \cdot 10^{-2}$ & $2.1 \cdot 10^{-2}$ & $2.1 \cdot 10^{-2}$ & $2.1 \cdot 10^{-2}$ & $2.1 \cdot 10^{-2}$ & $2.1 \cdot 10^{-2}$ \\
0.5 & $5.5 \cdot 10^{-3}$ & $8.7 \cdot 10^{-3}$ & $1.2 \cdot 10^{-2}$ & $2.6 \cdot 10^{-2}$ & $4.0 \cdot 10^{-2}$ & $4.1 \cdot 10^{-2}$ & $4.0 \cdot 10^{-2}$ & $4.0 \cdot 10^{-2}$ \\
0.7 & $1.8 \cdot 10^{-2}$ & $2.2 \cdot 10^{-2}$ & $1.5 \cdot 10^{-2}$ & $4.8 \cdot 10^{-3}$ & $8.9 \cdot 10^{-3}$ & $5.2 \cdot 10^{-2}$ & 0.10648 & 0.10507 \\
0.8 & $3.7 \cdot 10^{-2}$ & $4.4 \cdot 10^{-2}$ & $2.9 \cdot 10^{-2}$ & $1.9 \cdot 10^{-3}$ & $2.0 \cdot 10^{-2}$ & $1.4 \cdot 10^{-2}$ & 0.19175 & 0.14981 \\
0.9 & 0.07335 & $9.0 \cdot 10^{-2}$ & $6.5 \cdot 10^{-2}$ & $1.5 \cdot 10^{-2}$ & $4.1 \cdot 10^{-2}$ & $8.0 \cdot 10^{-2}$ & $1.6 \cdot 10^{-2}$ & 0.1997 \\
\hline
\end{tabular}

Example 4. Consider the nonlinear initial-boundary value problem

$$
\begin{aligned}
u_{t t}-t u_{x x} & =u^{2}-t^{2} x^{4}-2 t^{2} x^{3}-t^{2} x^{2}-2 t^{2}, \\
u(x, 0) & =0, \\
u_{t}(x, 0) & =x+x^{2}, \\
u(0, t) & =0 .
\end{aligned}
$$

The exact solution is $u=x t+x^{2} t$. We take $L=u_{t t}-u_{x x}$, and consequently

$$
\begin{aligned}
u_{0}= & \frac{1}{2} \int_{x-t}^{x+t}\left(y+y^{2}\right) d y=\frac{1}{3} t\left(t^{2}+3 x^{2}+3 x\right), \\
u_{0}= & \frac{1}{2} \int_{t-x}^{x+t}\left(y+y^{2}\right) d y=\frac{1}{3} x\left(3 t^{2}+3 t+x^{2}\right), \\
u_{1}= & \text { for } x>t, \\
u_{1} & =-\hbar\left(-\frac{1}{180} t^{8}+\frac{2}{45} t^{6} x^{2}+\frac{2}{45} t^{6} x-\frac{2}{3} t^{3}\right), \\
& +\frac{253}{36} t^{4} x^{4}-\frac{8}{9} t^{4} x^{3}-\frac{64}{9} t^{6} x^{2}+\frac{1}{45} t^{3} t^{5} x-\frac{23}{6} t^{3} x^{4}-\frac{1}{45} t^{3} x \\
& -\frac{1}{45} t^{2} x^{3}-\frac{35}{6} t^{2} x^{5}+t^{2} x^{2}-t^{2} x+\frac{1}{45} t x^{7}+\frac{71}{18} t x^{6} \\
& \left.+\frac{10}{3} t x^{3}-\frac{1}{360} x^{8}-\frac{125}{126} x^{7}-\frac{23}{6} x^{4}\right), \quad \text { for } x<t .
\end{aligned}
$$

The absolute errors between the exact and the two-term approximation of the series solution for some values of $(x, t) \in[0,1] \times[0,1]$ for $\hbar=-1$ are shown in Table 2. The exact solution for Example 4 is shown in the Figure 3 and the approximate solution is shown in Figure 4.

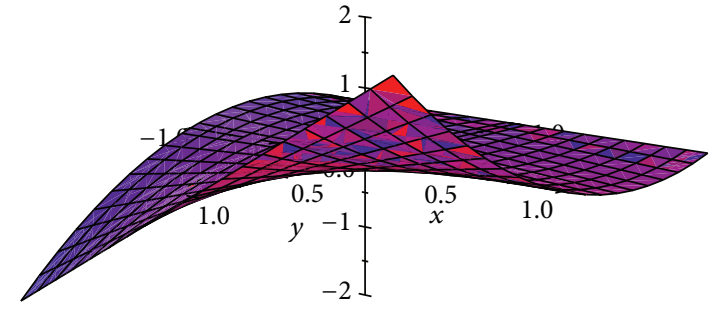

Figure 3: Exact solution for Example 4.

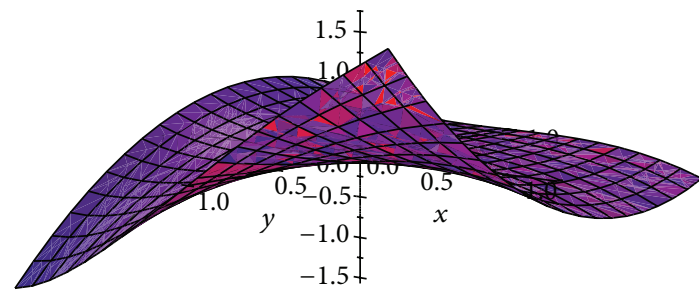

FIGURE 4: Approximate two-term approximation for Example 4.

Example 5. Consider the nonlinear initial-boundary value problem

$$
\begin{aligned}
u_{t t} & =\frac{6+(x-t)^{5}}{6} u_{x x}-u^{2}, \quad x>0, t>0, \\
u(x, 0) & =x^{3}, \\
u_{t}(x, 0) & =-3 x^{2}, \\
u(0, t) & =t^{3},
\end{aligned}
$$

whose exact solution is

$$
u= \begin{cases}(x-t)^{3} & \text { if } x>t, \\ (t-x)^{3} & \text { if } x<t .\end{cases}
$$

First we rewrite the equation as

$$
u_{t t}-u_{x x}=\frac{(x-t)^{5}}{6} u_{x x}-u^{2}
$$


The solution of the equation $u_{t t}-u_{x x}=0$ with the same initial-boundary conditions is

$$
\begin{aligned}
u_{0}= & \frac{1}{2}\left((x+t)^{3}+(x-t)^{3}\right)-\frac{1}{2} \int_{x-t}^{x+t} 3 y^{2} d y \\
= & (x-t)^{3}, \quad \text { for } x>t, \\
u_{0}= & \frac{1}{2}\left((x+t)^{3}-(t-x)^{3}\right)-\frac{1}{2} \int_{t-x}^{x+t} 3 y^{2} d y \\
& +(t-x)^{3}=(t-x)^{3}, \quad \text { for } x<t .
\end{aligned}
$$

For $u_{1}$ we have

$$
\begin{aligned}
& L\left(u_{1}\right)=-\hbar\left[\frac{(x-t)^{5}}{6} u_{0 x x}-u_{0}^{2}\right], \\
& u_{1}=-\frac{\hbar}{2} \int_{0}^{t} \int_{x-t+s}^{x+t-s}\left(\frac{(y-s)^{5}}{6} 6(y-s)-(y-s)^{6}\right) d y d s \\
& =0, \quad \text { for } x>t \text {, } \\
& u_{1}=-\frac{\hbar}{2} \int_{0}^{t-x} \int_{t-x-s}^{x+t-s}\left(\frac{(s-y)^{5}}{6} 6(s-y)-(s-y)^{6}\right) d y d s \\
& -\frac{\hbar}{2} \int_{t-x}^{t} \int_{x-t+s}^{x+t-s}\left(\frac{(s-y)^{5}}{6} 6(s-y)-(s-y)^{6}\right) d y d s \\
& =0
\end{aligned}
$$

for $x<t$, and for the exact solution we obtain $u=u_{0}+u_{1}$.

\section{Discussion}

The main goal of this work was to propose a reliable method for solving wave-like equations with variable coefficients. The proposed equations may not be solved by the method of separation of variables or by HAM or ADM in standard form. The main difficulty in the use of previous methods is related to the choice of an auxiliary linear operator $L$. Traditional methods work effectively in case of analytical solutions in the whole region, that is, in fact, when some initial/boundary condition is supposed by another one or in case of some special type of equations (homogeneous, etc.).

The proposed method was applied directly without any need for restrictive assumptions, and this gives it a wider applicability. This method is capable of greatly reducing the volume of computational work compared to standard approaches while still maintaining high accuracy of the approximate solution. A higher accuracy level can be attained even by evaluating some two-three terms in the series solution.

The approach was tested by employing the method to obtain solutions for several problems. The results obtained in all cases demonstrate the efficiency of this approach.

\section{Conflict of Interests}

The author declares that there is no conflict of interests regarding the publication of this paper.

\section{References}

[1] P. J. Caudrey, J. C. Eilbeck, and J. D. Gibbon, “The sine-Gordon equation as a model classical field theory," Il Nuovo Cimento B, vol. 25, no. 2, pp. 497-512, 1975.

[2] R. K. Dodd, I. C. Eilbeck, and J. D. Gibbon, Solitons and Nonlinear Wave Equations, Academic Press, London, UK, 1982.

[3] J. R. Holliday, J. B. Rundle, K. F. Tiampo, W. Klein, and A. Donnellan, "Modification of the pattern informatics method for forecasting large earthquake events using complex eigenfactors," Tectonophysics, vol. 413, no. 1-2, pp. 87-91, 2006.

[4] A. A. Akhmetov, "Long current loops as regular solutions of the equation for coupling currents in a flat two-layer superconducting cable," Cryogenics, vol. 43, no. 3-5, pp. 317-322, 2003.

[5] G. D. Manolis and T. V. Rangelov, "Non-homogeneous elastic waves in soils: notes on the vector decomposition technique," Soil Dynamics and Earthquake Engineering, vol. 26, no. 10, pp. 952-959, 2006.

[6] A. R. Manwell, The Tricomi Equation with Applications to the Theory of Plane Transonic Flow, Pitman, Marshfield, Mass, USA, 1979.

[7] A. V. Bitsadze, Some Classes of Partial Differential Equations, Gordon \& Breach, 1988, (Russian).

[8] L. D. Landau and E. M. Lifshitz, Fluid Mechanics, Pergamon Press, 1982.

[9] A.-M. Wazwaz, "The tanh method: exact solutions of the sineGORdon and the sinh-GORdon equations," Applied Mathematics and Computation, vol. 167, no. 2, pp. 1196-1210, 2005.

[10] D. Kaya, "A numerical solution of the sine-Gordon equation using the modified decomposition method," Applied Mathematics and Computation, vol. 143, no. 2-3, pp. 309-317, 2003.

[11] A. Aslanov, "The homotopy-perturbation method for solving klein-gordon-type equations with unbounded right-hand side," Zeitschrift für Naturforschung Section A, vol. 64, no. 1-2, pp. 149152, 2009.

[12] S. M. El-Sayed, "The decomposition method for studying the Klein-Gordon equation," Chaos, Solitons \& Fractals, vol. 18, no. 5, pp. 1025-1030, 2003.

[13] A.-M. Wazwaz and A. Gorguis, "Exact solutions for heat-like and wave-like equations with variable coefficients," Applied Mathematics and Computation, vol. 149, no. 1, pp. 15-29, 2004.

[14] S. J. Liao, The proposed homotopy analysis technique for the solution of nonlinear problems [Ph.D. thesis], Shanghai Jiao Tong University, Shanghai, China, 1992.

[15] S.-J. Liao, "An approximate solution technique not depending on small parameters: a special example," International Journal of Non-Linear Mechanics, vol. 30, no. 3, pp. 371-380, 1995.

[16] S.-J. Liao, "A kind of approximate solution technique which does not depend upon small parameters-II. An application in fluid mechanics," International Journal of Non-Linear Mechanics, vol. 32, no. 5, pp. 815-822, 1997.

[17] S. J. Liao, Beyond Perturbation: Introduction to the Homotopy Analysis Method, Chapman \& Hall, CRC, Boca Raton, Fla, USA, 2003.

[18] S. J. Liao, "An optimal homotopy-analysis approach for strongly nonlinear differential equations," Communications in Nonlinear Science and Numerical Simulation, vol. 15, no. 8, pp. 2003-2016, 2010.

[19] T. M. Hayat and M. Sajid, "On analytic solution for thin film flow of a fourth grade fluid down a vertical cylinder," Physics Letters A: General, Atomic and Solid State Physics, vol. 361, no. 4-5, pp. 316-322, 2007. 
[20] S. Abbasbandy, "The application of homotopy analysis method to nonlinear equations arising in heat transfer," Physics Letters A, vol. 360, no. 1, pp. 109-113, 2006.

[21] A. Aslanov, "Homotopy perturbation method for solving wavelike nonlinear equations with initial-boundary conditions," Discrete Dynamics in Nature and Society, vol. 2011, Article ID 534165, 10 pages, 2011.

[22] R. Rajaraman, "Analytical solutions for some of the nonlinear hyperbolic-like equations with variable conditions," Global Journal of Science Frontier Research Mathematics and Decision Sciences, vol. 12, no. 5, pp. 54-60, 2012.

[23] A. K. Alomari, M. S. M. Noorani, and R. Nazar, "Solutions of heat-like and wave-like equations with variable coefficients by means of the homotopy analysis method," Chinese Physics Letters, vol. 25, no. 2, pp. 589-592, 2008.

[24] T. Öziş and D. Ağırseven, "He’s homotopy perturbation method for solving heat-like and wave-like equations with variable coefficients," Physics Letters A, vol. 372, no. 38, pp. 5944-5950, 2008.

[25] E. M. E. Zayed, T. A. Nofal, and K. A. Gepreel, “The homotopy perturbation method for solving nonlinear Burgers and new coupled MKdV equations," Zeitschrift für Naturforschung A, vol. 63, pp. 627-633, 2008.

[26] E. M. E. Zayed, T. A. Nofal, and K. A. Gepreel, "Homotopy perturbation and Adomain decomposition methods for solving nonlinear Boussinesq equations," Communications on Applied Nonlinear Analysis, vol. 15, no. 3, pp. 57-70, 2008.

[27] E. M. E. Zayed, T. A. Nofal, and K. A. Gepreel, "On using the homotopy perturbation method for finding the traveling wave solutions of generalized nonlinear Hirota-Satsuma coupled $\mathrm{KdV}$ equations," International Journal of Nonlinear Science, vol. 7, no. 2, pp. 159-166, 2009.

[28] E. M. Zayed, T. A. Nofal, and K. A. Gepreel, "The travelling wave solutions for non-linear initial-value problems using the homotopy perturbation method," Applicable Analysis, vol. 88, no. 4, pp. 617-634, 2009.

[29] E. M. E. Zayed and H. M. Abdel-Rahman, "The homotopy perturbation method and the Adomian decomposition method for the nonlinear coupled equations," Journal of Partial Differential Equations, vol. 22, no. 4, pp. 334-351, 2009.

[30] E. M. E. Zayed and H. M. Abdel Rahman, "The variational iteration method and the variational homotopy perturbation method for solving the KdV-Burgers equation and the SharmaTasso-Olver equation," Zeitschrift für Naturforschung A, vol. 65, no. 1, pp. 25-33, 2010.

[31] E. M. E. Zayed and H. M. Abdel Rahman, "The homotopy analysis method for solving the nonlinear evolution equations in mathematical physics," Communications on Applied Nonlinear Analysis, vol. 18, no. 3, pp. 53-70, 2011.

[32] W. A. Strauss, Partial Differential Equations, John Wiley \& Sons, New York, NY, USA, 1992. 


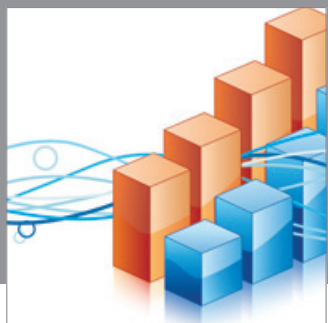

Advances in

Operations Research

mansans

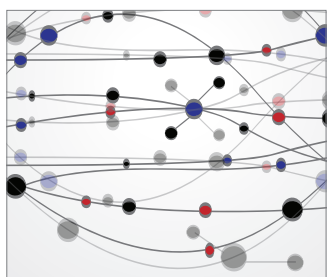

The Scientific World Journal
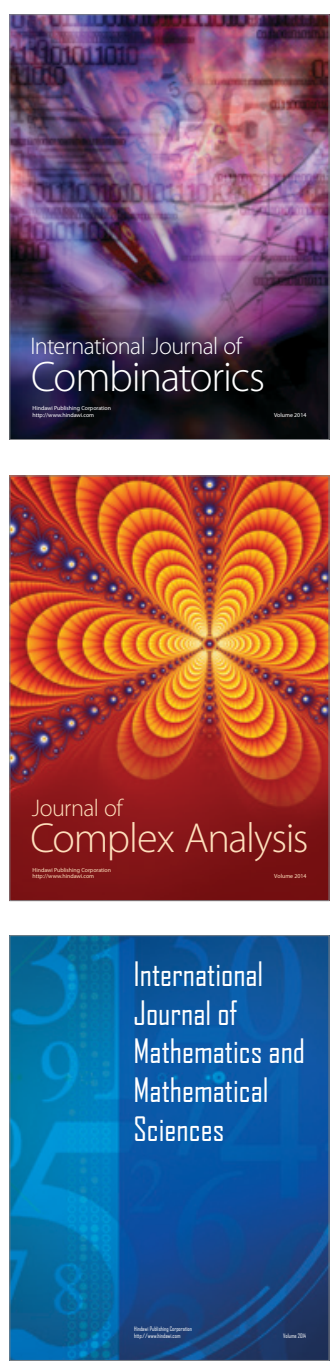
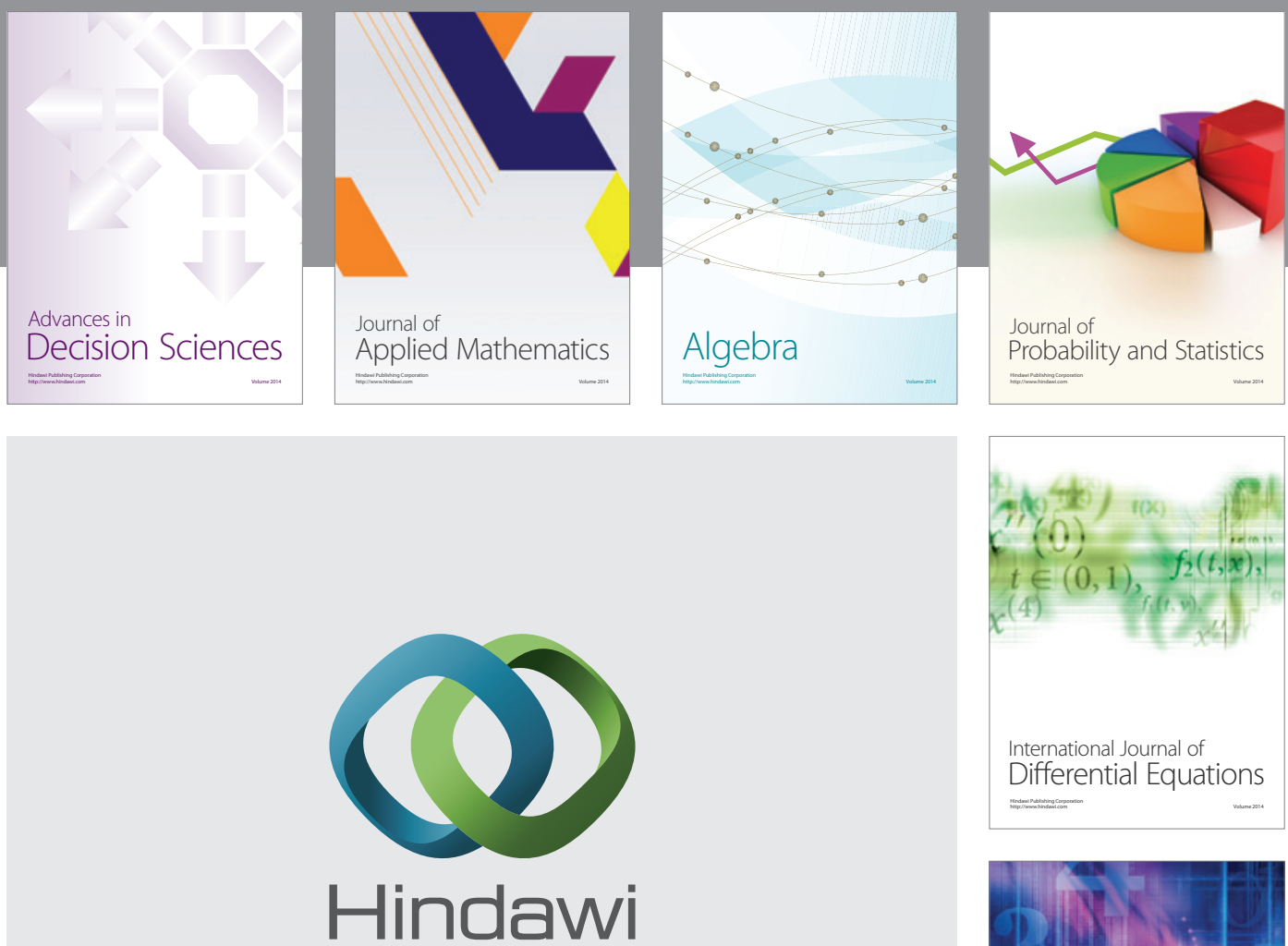

Submit your manuscripts at http://www.hindawi.com
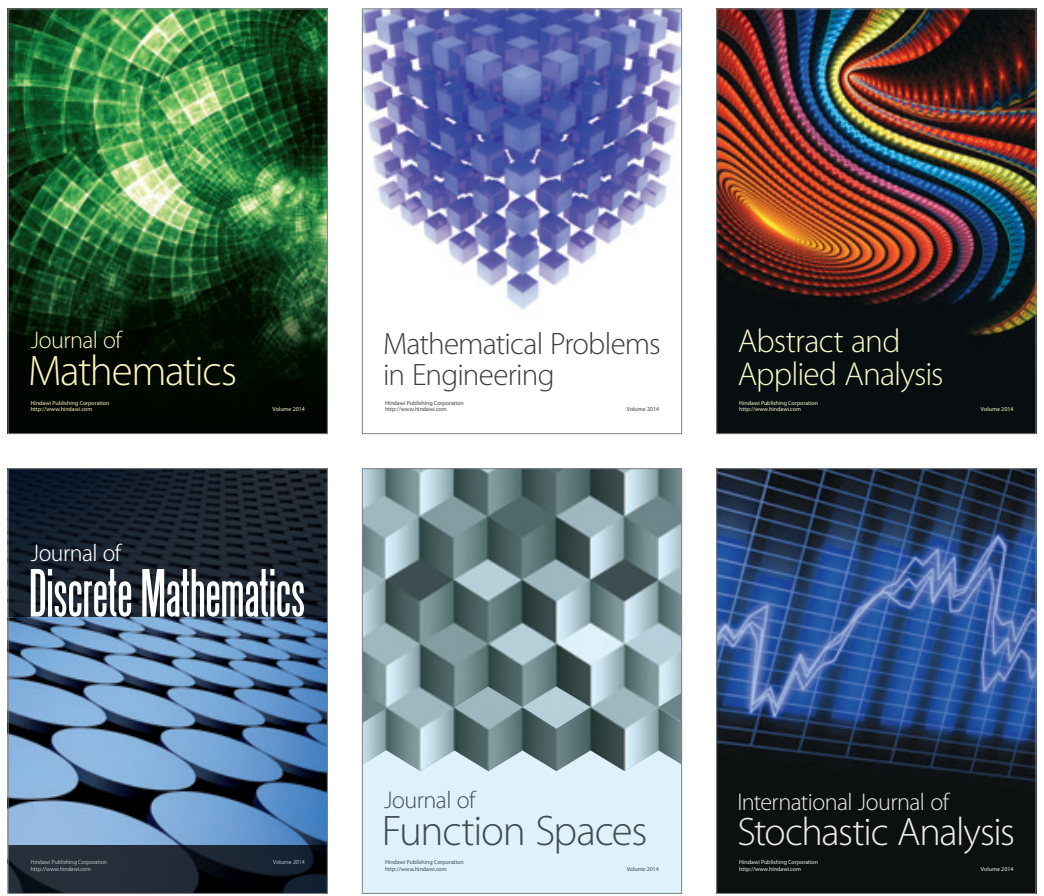

Journal of

Function Spaces

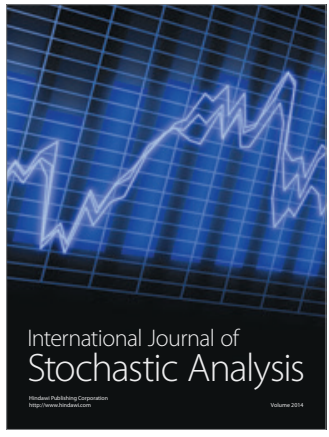

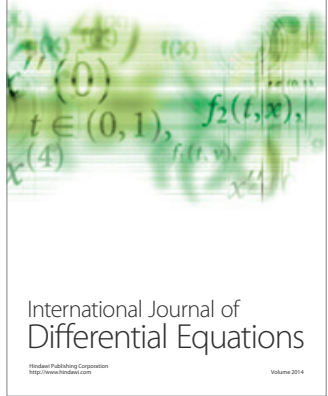
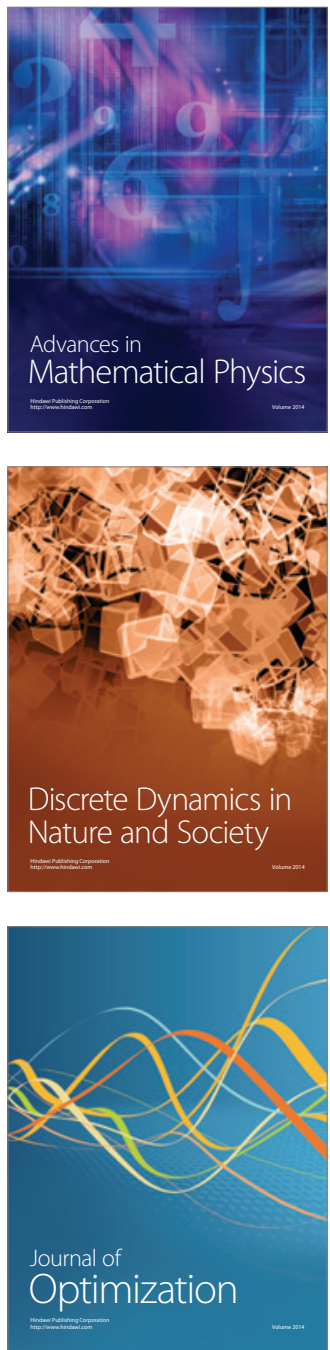